\title{
Design and Performance of the Alignment System for the CMS Muon Endcaps
}

Marcus Hohlmann, Member, IEEE, Gyöngyi Baksay, Max Browngold, Klaus Dehmelt, Samir Guragain,Valery Andreev, Xiaofeng Yang, James Bellinger, Duncan Carlsmith, Farshid Feyzi, Richard J. Loveless, David Northacker, Michael Case, David P. Eartly, Oleg Prokofiev, Vladimir Sknar, Valeri Sytnik

\begin{abstract}
The alignment system for the CMS Muon Endcap detector employs several hundred sensors such as optical 1-D CCD sensors illuminated by lasers and analog distance- and tiltsensors to monitor the positions of one sixth of 468 large Cathode Strip Chambers. The chambers mounted on the endcap yoke disks undergo substantial deformation on the order of centimeters when the 4T field is switched on and off. The Muon Endcap alignment system is required to monitor chamber positions with 75-200 $\mu \mathrm{m}$ accuracy in the $R \varphi$ plane, $\sim 400 \mu \mathrm{m}$ in the radial direction, and $\sim 1 \mathrm{~mm}$ in the z-direction along the beam axis. The complete alignment hardware for one of the two endcaps has been installed at CERN. A major system test was performed when the $4 \mathrm{~T}$ solenoid magnet was ramped up to full field for the first time in August 2006. We present the overall system design and first results on disk deformations, which indicate that the measurements agree with expectations.
\end{abstract}

\section{INTRODUCTION}

$\mathrm{I}_{\mathrm{n}}$ In each of the two CMS muon endcaps [1], 234 large cathode strip chambers (CSCs) with sizes up to $3.4 \mathrm{~m} \times 1.5 \mathrm{~m}$ are mounted in 4 stations on large iron yoke disks [2] (Fig. 1). Fig. 2 shows one such station. The Muon Endcap (ME) alignment system is organized around the need to continuously and accurately monitor the actual position of each of the 468 CSCs relative to the Tracking System. Due to the large magnetic field, the chambers mounted on the endcap yokes undergo substantial motion and deformation on the order of centimeters when the field is switched on and off. Fig. 3 shows an overview of components in the Muon Endcap Alignment System as implemented in a simulation of the

Manuscript received November 17, 2006. This work was supported in part by the U.S. Department of Energy under Grant Nos. DE-AC02-76-CH03000 and DE-FG02-03ER41264 A005.

M. Hohlmann, G. Baksay, M. Browngold, K. Dehmelt, and S. Guragain are with the Department of Physics and Space Sciences, Florida Institute of Technology, Melbourne, FL, USA (telephone: 321-674-7275, e-mail: hohlmann@fit.edu).

V. Andreev and X.Yang are with the Department of Physics, University of California, Los Angeles, CA, USA.

J. Bellinger, D. Carlsmith, F. Feyzi, R. J. Loveless, and D. Northacker are with the Department of Physics, University of Wisconsin, Madison, WI, USA.

M. Case is with the Department of Physics, University of California, Davis, CA, USA.

D. P. Eartly and O. Prokofiev are with the Fermi National Accelerator Lab, Batavia, IL, USA.

V. Sknar is with the Petersburg Nuclear Physics Institute, St. Petersburg, Russia.

V. Sytnik is with the Department of Physics, University of California, Riverside, CA, USA

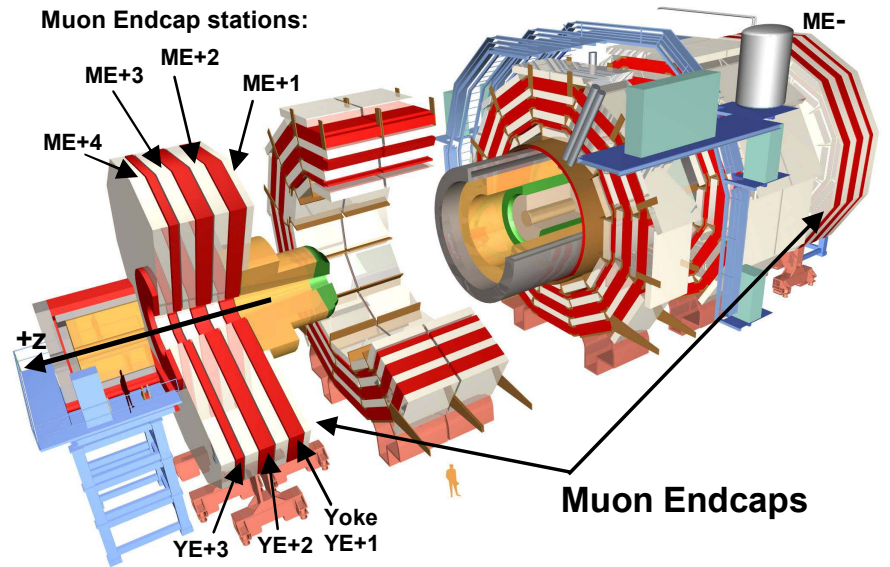

Fig. 1. General layout of the muon system in the CMS Detector.

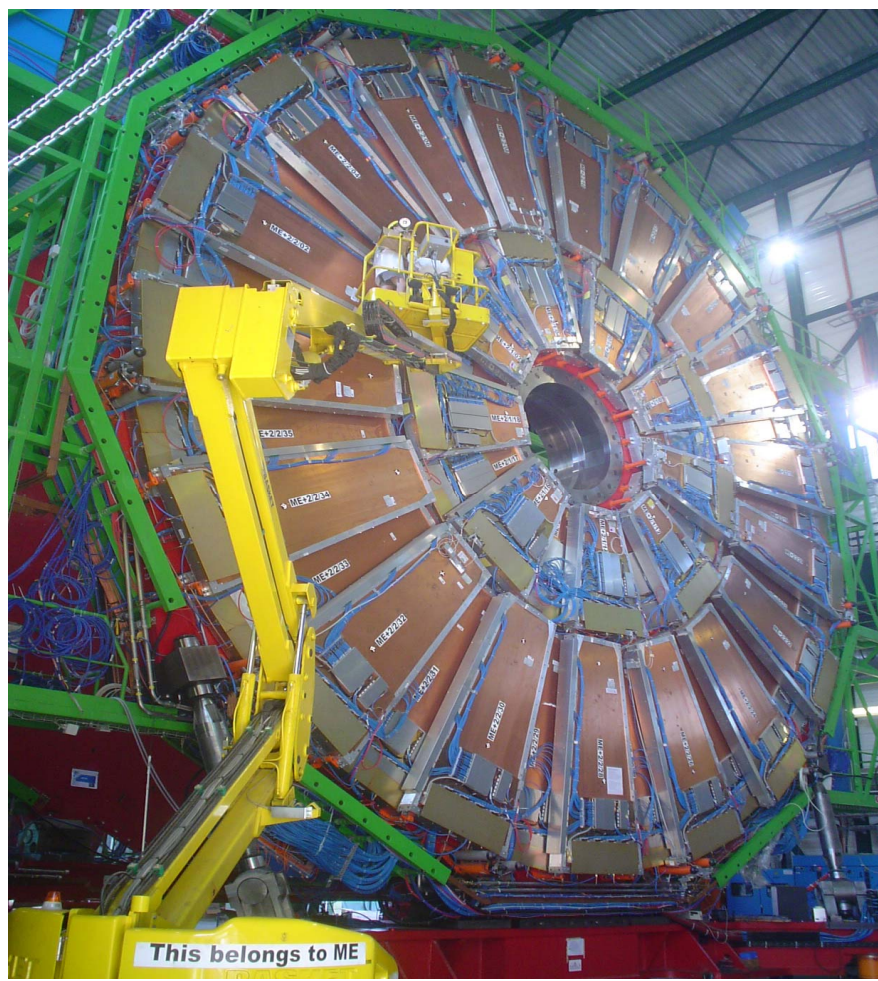

Fig. 2. Fully instrumented CMS Muon Endcap station ME+2. Note the inner and outer rings of cathode strip chambers with overlaps between chambers in $\varphi$. 
system geometry. The ME Alignment System is part of the overall Muon Alignment effort in CMS, which also includes the Muon Barrel Alignment and a Link to the Central Tracker.

\section{AlignMENT REQUIREMENTS}

The task of the ME Alignment is to provide references for the positions of CSCs relative to each other, relative to the Central Si-Tracker, and ultimately within the absolute coordinates of CMS. The alignment system must measure and monitor the absolute positions of the CSCs in the $\mathrm{R} \varphi$ plane, and in $\mathrm{Z}$.

\section{A. $R \varphi$ alignment}

The $R \varphi$ resolution requirement for the CSCs and hence for the alignment requirement is mainly constrained by the multiple scattering of muons along their tracks through the endcaps. From simulations, the requirements on the absolute alignment accuracy were found to be $75 \mu \mathrm{m}$ for the middle ring of ME1 chambers and $200 \mu \mathrm{m}$ for the other CSC's [3]. The required accuracy in the R-position measurement is dictated by the $\varphi$ alignment requirements, as there is a direct coupling between $\mathrm{R}$ alignment and $\mathrm{R} \varphi$ accuracy. From simulations it is found to be $\sim 400 \mu \mathrm{m}$.

\section{B. Z alignment}

The total $\mathrm{Z}$ displacements due to the deformation of the iron yoke disks caused by the strong and non-uniform magnetic field in the endcaps was expected to be $1-2 \mathrm{~cm}$ based on finite element analysis of the yokes. The alignment sensors need to be able to accommodate that dynamic range with an accuracy of $1 \mathrm{~mm}$ or less.

\section{System Design}

The ME alignment uses a complex arrangement of five types of sensors for the transferring and monitoring of $\varphi, \mathrm{R}$, and $\mathrm{Z}$ coordinates as shown in Fig. 3. Forced by CMS geometry constraints and economics, the system aligns only one sixth of all chambers. The main monitoring tools within the $R \varphi$ plane are Straight Line Monitors (SLMs) such as the one sketched in Fig. 4. Two crosshair lasers, which emit a nearly radial laser beam across four chambers from each end, provide straight reference lines that are picked up by two optical sensors (Digital CCD Optical Position Sensors, DCOPS, [4]) on each chamber. This arrangement provides references for the chamber positions relative to the laser lines.

Fig. 5 shows a photograph of a complete SLM on station $\mathrm{ME}+2$. The figure indicates also R-sensors for monitoring radial chamber positions, Z-sensors for axial distance measurements between stations, and a clinometer for monitoring the tilt of the transfer plate onto which lasers, reference DCOPS, and Z-sensors are mounted. The inset in Fig. 5 shows the location of proximity sensors in the outer ring of the ME+1 station, which monitor the azimuthal distances between neighboring chambers. These are necessary because

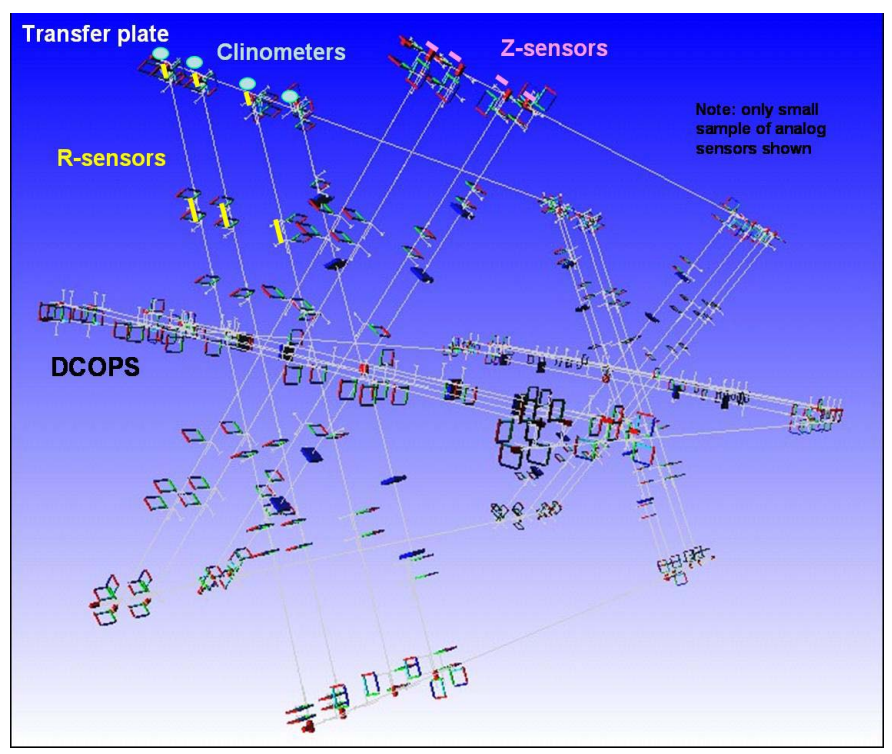

Fig. 3. Visualization of the geometry and components of the CMS Muon Endcap Alignment system. The square objects represent optical sensors (DCOPS) for monitoring 3 straight laser lines across each muon endcap station.

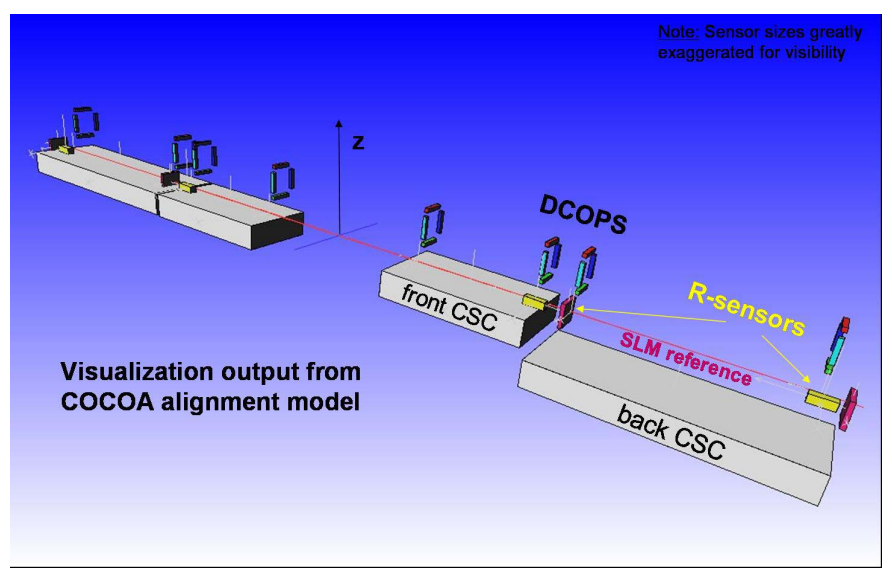

Fig. 4. Sketch of Straight Line Monitor (SLM) with optical (DCOPS) and analog radial sensors. Actual chamber shapes are wedges, not rectangles.

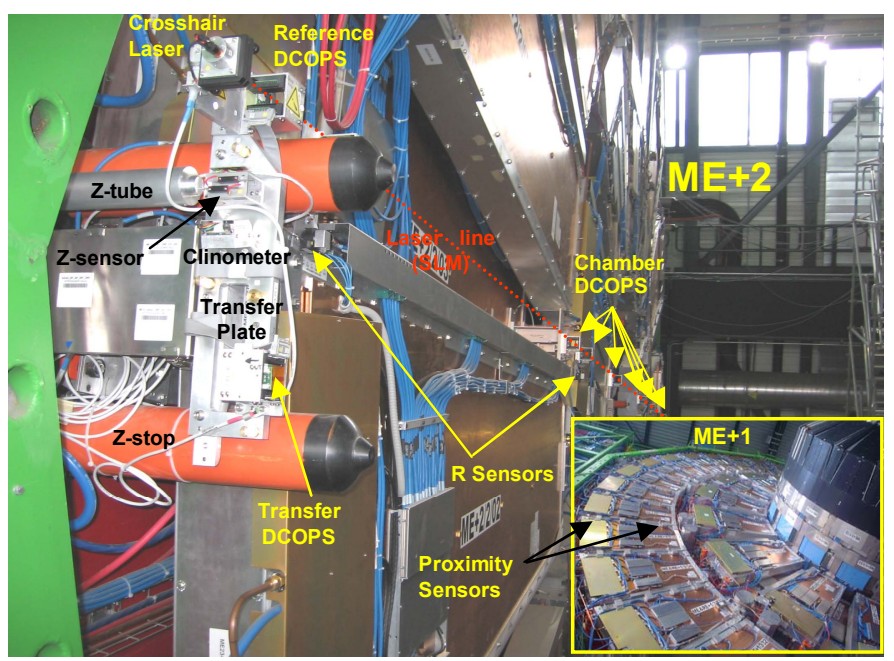

Fig. 5. Close-up of one of the three Straight Line Monitors (SLM) on the $\mathrm{ME}+2$ station with crosshair laser, DCOPS, and analog sensors (R, Z, Clinometer). The inset indicates the locations of Proximity sensors on ME +1 . 
the outer ring of chambers on $\mathrm{ME}+1$ is the only ring of chambers in which the CSCs do not overlap in $\varphi$.

Finally, every CSC and Transfer plate is equipped with photogrammetry targets to allow absolute magnet-off measurements. Subsequent alignments for magnet-on conditions, however, must rely on monitoring all positions with the system of optical sensors and analog sensors.

\section{A. Azimuthal Chamber Position Monitoring}

The $\varphi$ coordinate alignment is handled by optical SLMs and transfer lines. Transfer laser lines run parallel to the CMS Zaxis along the outer cylindrical envelope of CMS at six points separated by 60 degrees in $\varphi$. The SLMs run across the surfaces of one sixth of all the CSCs, along radial directions, and link two Transfer lines on opposite sides of a disk.

Both laser lines have similar basic configurations: a laser beam defines a direction in space that is picked up by several DCOPS precisely mounted on CSCs or Transfer Plates to reference their own positions. Mounting accuracies due to tolerances of dowel pins and dowel holes are on the order of $50 \mu \mathrm{m}$. Every DCOPS comprises 4 linear CCDs - each with 2048 pixels and $14 \mu \mathrm{m}$ pixel pitch. The CCDs are basically arranged in the shape of a square and can be illuminated by crosshair lasers from either side as seen in Fig. 5. In case of an SLM, this allows simultaneous monitoring of the $\varphi$ and $Z$ coordinates with two CCDs for each coordinate. Each coordinate is measured twice for redundancy and also for monitoring any chamber rotations around the SLM axis. An azimuthal chamber displacement can then be detected by observing a corresponding shift in the position of laser beam profiles across the CCDs.

\section{B. Radial Chamber Position Monitoring}

The $\mathrm{R}$ coordinate on an outer chamber under an SLM is determined with respect to the ME Transfer plate. A linear wire-extension potentiometer measures the radial distance between that outer chamber and the Transfer plate. Another potentiometer monitors the radial distance between an outer and inner chamber (Figs. 4 and 5). Absolute radial chamber positions are measured initially by photogrammetry of SLMs across the disks at $\mathrm{B}=0 \mathrm{~T}$. Only the $\mathrm{R}$ measurements between CSCs performed by analog R-sensors can provide $\mathrm{R}$ information after the initial survey and photogrammetry

\section{Axial Referencing of Chambers}

The absolute $\mathrm{Z}$ coordinate for each station is transferred from the Muon Barrel to the Muon Endcap Transfer plates at six points around the station in azimuth. This is done with axial aluminum tubes of calibrated length that on each end are in contact with fixed linear-motion potentiometers (Z-sensors, see Fig. 5) on the Transfer plates. IR-laser distancemeters attached to and extending from the ME+1 Transfer plates reference surfaces on the Muon Barrel yoke at six points to link Muon Endcap and Muon Barrel in Z.

\section{Monitoring of Transfer plate tilts}

Lasers, reference DCOPS's, and transfer DCOPS's are mounted on the Transfer plates. Any tilt of these plates away from their nominal positions changes the direction of the SLM laser beam and introduces a bias in the measurements of the Transfer DCOPS. Consequently, the orientations of the Transfer plates must be carefully monitored with clinometers.

These clinometer sensors measure tilts in 2 perpendicular directions by electronically monitoring the capacitance of a small plastic cavity filled with a fluid. They are mounted in brackets on the Transfer plates (Fig. 5) so that the reference plane of the sensor is always horizontal and thus parallel to the fluid level - irrespective of the spatial orientation of the Transfer plates.

\section{E. Proximity measurements between ME1 chambers}

The proximity sensors in the outer ring of the $\mathrm{ME}+1$ station monitor the azimuthal distances between neighboring chambers near their inner and outer radii. Each chamber is referenced to its immediate two neighbors and carries two proximity sensors (see inset in Fig. 5). The sensor used for this application is a linear-motion potentiometer of the same type as used for the Z-sensors. The sensor is mounted on one chamber and the potentiometer's linear-motion rod is in mechanical contact with a neighboring chamber.

\section{SEnsor CALibrations}

Analog sensors were calibrated with a 1-D precision linear mover with $6.4 \mu \mathrm{m}$ step size, which can be used for extending the wire of an R-sensor, moving the rod of a linear-motion potentiometer, tilting a clinometer, or reflecting the beam from an IR-laser Z-sensor. The total uncertainty in the absolute distance calibration is $100 \mu \mathrm{m}$ for $\mathrm{R}$ sensors and $53 \mu \mathrm{m}$ for $\mathrm{Z}$ sensor types [5], which is well within the range of the requirements.

Calibration for optical DCOPS's consisted in determining the distance from the surface of the mount hole for a reference dowel pin to the first active CCD pixel and measuring the projected pixel pitch of each of the four CCDs. This was done on a calibration bench where a fiber bundle variable light source at the focus of a parabolic mirror illuminated a mask with eight optical slits. For calibration a sensor was located behind the mask so that each CCD was illuminated by two slits. Behind the sensor to be calibrated was a permanently mounted DCOPS that assured the stability of the system through consistency in repeated measurements and calibrations over time.

Typically a set of 16 test beam profiles were recorded. A profile with two peaks and a background were fitted for each measurement. The results were averaged. A simple geometry reconstruction based on coordinate-measuring-machine data for the calibration mask and sensor mounts determined the physical pixel positions. For a given mounting, calibrations repeated within a pixel. However, calibration errors in repeated mountings on the dowels were larger; typically 30 to $50 \mu \mathrm{m}$ due to fit tolerances. 


\section{Performance Results From Magnet Test}

A crucial test of the large superconducting solenoid magnet in the CMS detector was successfully performed between June and November 2006. Stable operation at full field (4T) was achieved. One muon endcap has been fully instrumented with alignment sensors for this test. The sensors, readout, and DAQ software were commissioned during this test period. This allowed the first full-scale test of one of the main tasks of the alignment system, i.e. monitoring of the expected substantial movement of an endcap yoke disk with mounted CSCs when the magnetic field is ramped up to its maximum value of $4 \mathrm{~T}$.

The data presented here were taken over a period of three days (Aug. 26-28, 2006) during which the magnet was ramped up in several steps from 0T to full field in several steps as shown in Fig. 6.

\section{A. DCOPS Performance}

Typical laser beam profiles measured by the 4 CCDs in a representative DCOPS are shown in Fig. 7. The typical uncertainty in the centroid position from a Gaussian plus quadratic fit to such a beam profile shape is $\sim 0.2$ pixels or $\sim 3$ $\mu \mathrm{m}$. The z-position of a crosshair laser is calculated from a fit of an SLM with 4 DCOPS in ME+1. The displacement of the laser in $\mathrm{Z}$ vs. magnetic field shown in Fig. 8 clearly indicates a deformation of the yoke disk.

\section{B. R-Sensor Results}

The radial displacements of outer chambers relative to the Transfer plates on the outer edges of the disks were generally found to be considerably less than $50 \mu \mathrm{m}$. Radial distances between inner and outer chambers at the six monitoring points around each of stations $\mathrm{ME}+1,+2,+3$ are larger and are shown as a function of the central magnetic field in Fig. 9. Maximum radial chamber displacements at full field are about $500 \mu \mathrm{m}$ for $\mathrm{ME}+1$, and on the order of 100 to $200 \mu \mathrm{m}$ for $\mathrm{ME}+2$ and $\mathrm{ME}+3$.

The signs of the various measured displacements $\Delta \mathrm{R}$ indicate that the side of the endcap disk $(\mathrm{YE}+1)$ facing the barrel and carrying the $\mathrm{ME}+1$ chambers (neg. $\Delta \mathrm{R}$ ), gets compressed. In contrast, the front face of the next yoke disk $(\mathrm{YE}+2)$ carrying the $\mathrm{ME}+2$ chambers (pos. $\Delta \mathrm{R}$ ) expands, whereas the back side of $\mathrm{YE}+2$, which carries the $\mathrm{ME}+3$ chambers, compresses (neg. $\Delta \mathrm{R}$ ).

\section{Z-Sensor Results}

The axial distances between the ME +1 station and the Muon Barrel were monitored with IR-laser distancemeters for three points around the ME +1 disk. (The other three points could not be instrumented during the test.) Results of these measurements are shown in Fig. 10. They track each other and the variations in the central magnetic field well (Fig. 6).

The positive sign of the measured displacements indicates a bending of the $\mathrm{YE}+1$ disk away from the Muon Barrel around the outer edge on the order of 3 to $6 \mathrm{~mm}$. Fig. 11 shows the axial displacement of the outer perimeter of ME +1 vs. central magnetic field. A quadratic dependence on the magnetic field

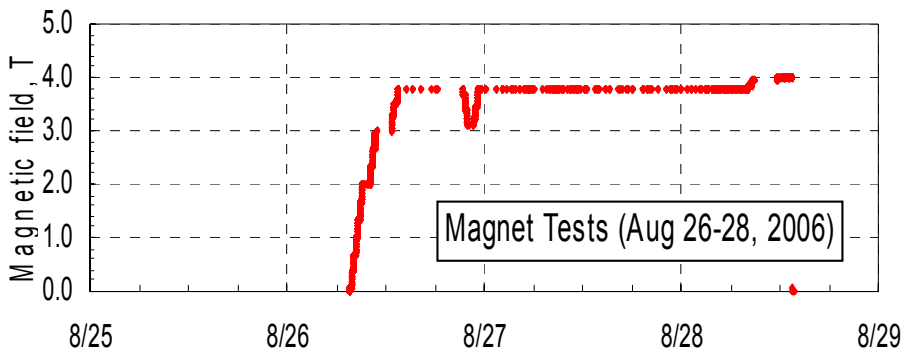

Fig. 6. Central B-field in the CMS solenoid during alignment test period.
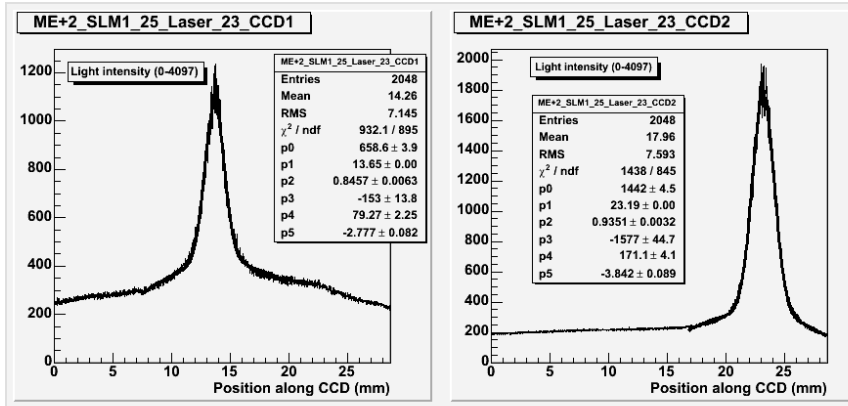

ME+2_SLM1_25_Laser_23_CCD3
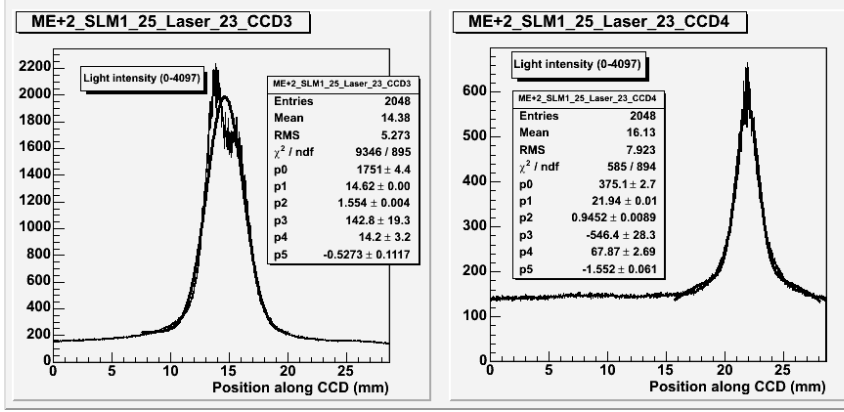

Fig. 7. The laser intensity (in arbitrary units) vs. position for typical laser beam profiles as measured with the 4 CCDs in a representative DCOPS during the Magnet Test. Fits are Gaussian plus a quadratic function.
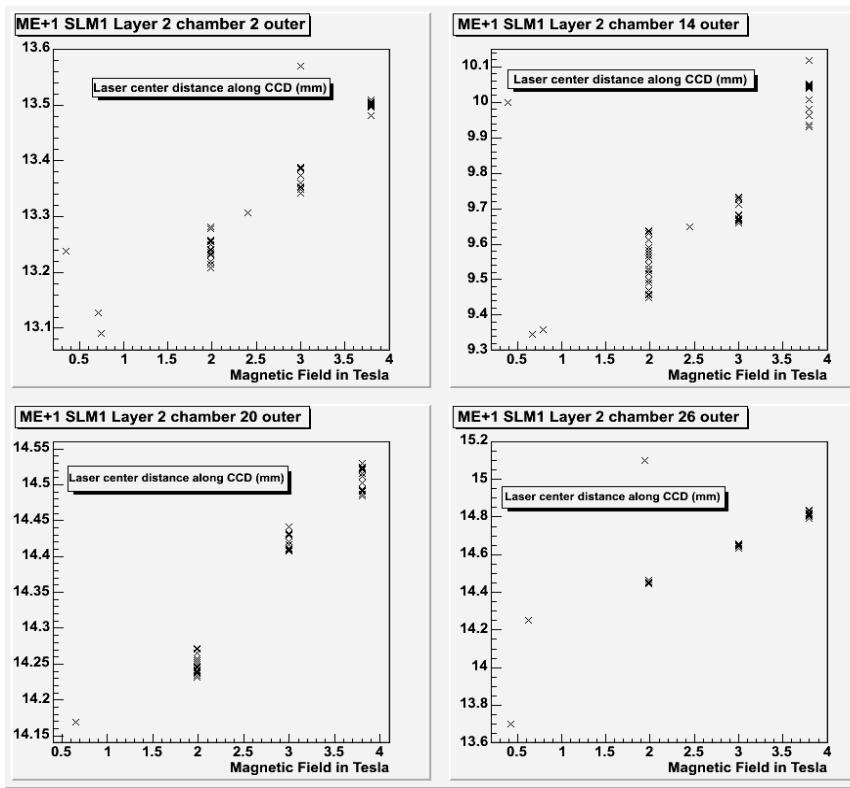

Fig. 8. Z-positions of crosshair lasers (in $\mathrm{mm}$ ) measured with 4 DCOPS vs. magnetic field for an SLM on ME+1. The observed slopes indicate a deformation of the yoke with magnetic field. 
is apparent, which is in agreement with expectations from Finite Element Analysis of disk deformations.

\section{Clinometer Results}

The back bending of the chambers at the outer perimeter was also detected with the clinometers, which are mounted on the Transfer plates near the Z-sensors. The back bending is basically azimuthally symmetric around the disk due to the cylindrical symmetry of the solenoidal magnetic field that causes it. As the clinometers must always be mounted horizontally, i.e. parallel to the ground floor, their sensitivity to the back bending varies sinusoidally around the perimeter of the disk. The expected behavior is shown in Fig. 12 as a dashed red curve.

The data basically follow the expected sinusoidal behavior for all four $\mathrm{ME}$ stations with a maximum bending angle relative to the vertical of $\sim 2.5 \mathrm{mrad}$ (Fig. 12). At the lower part of the disk (near point 5) the bending is slightly less because here the disks are more confined by the support carts on which the disks are mounted.

Station $\mathrm{ME}+1$ is not only equipped with clinometers at the outer edge on the Transfer plates, but also on six middle-ring chambers in the (half) SLMs near their inner edges. The measured larger bending angle at the inner radius as shown in
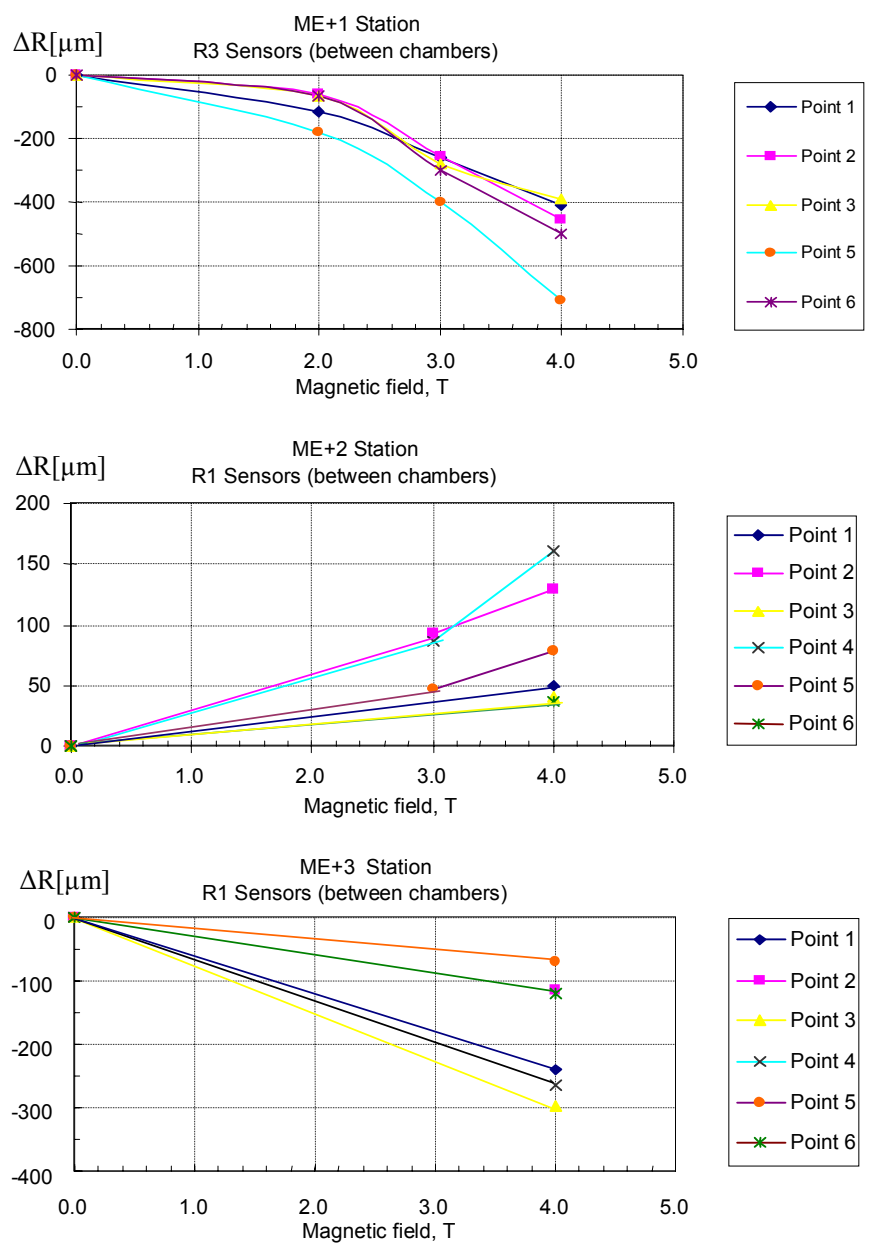

Fig. 9. Radial displacements $\Delta \mathrm{R}$ between six pairs of CSC chambers at each of stations $\mathrm{ME}+1,+2,+3$ vs. central magnetic field.
Fig.13 is consistent with independent displacement measurements by the Link alignment system and indicates increased bending (bulging) of the $\mathrm{YE}+1$ disk inside the array of Z-stop standoffs between the Muon Barrel and Endcap disks.

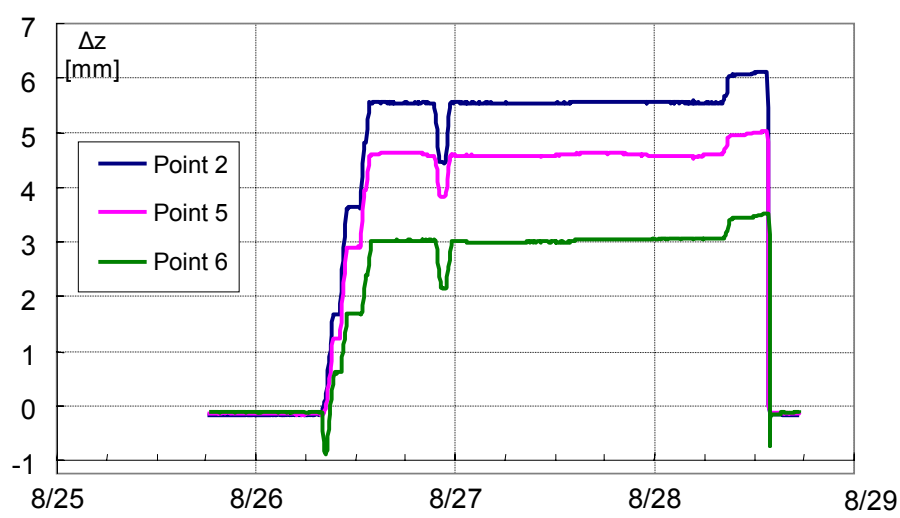

Fig. 10. Displacement $\Delta \mathrm{z}$ in $\mathrm{z}$ direction measured for the outer perimeter of the ME +1 disk with IR-laser $Z$-sensors at 3 different points during the test.

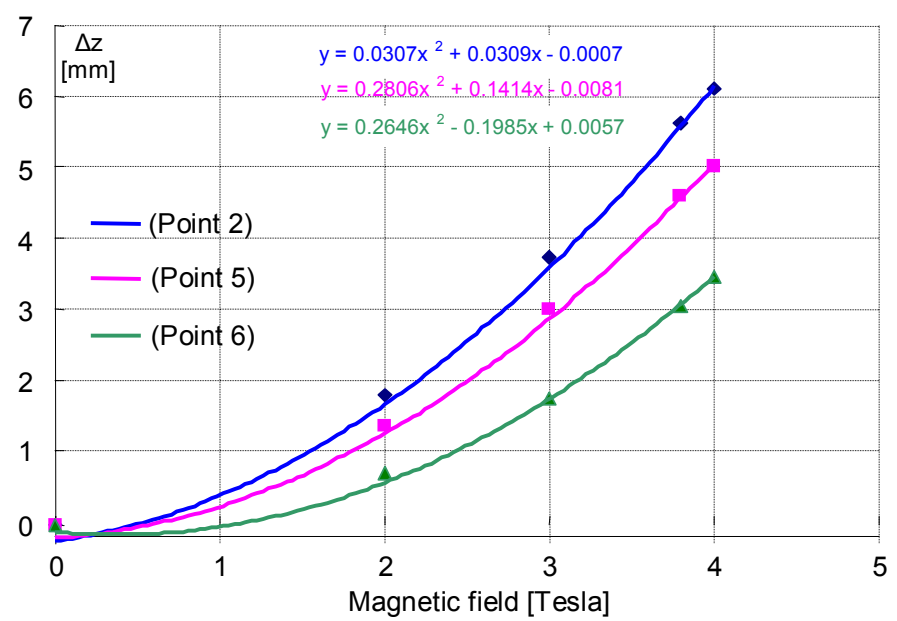

Fig. 11. Displacement $\Delta z$ vs. central magnetic field measured for three different points at the outer perimeter of the $\mathrm{ME}+1$ station and quadratic fits.

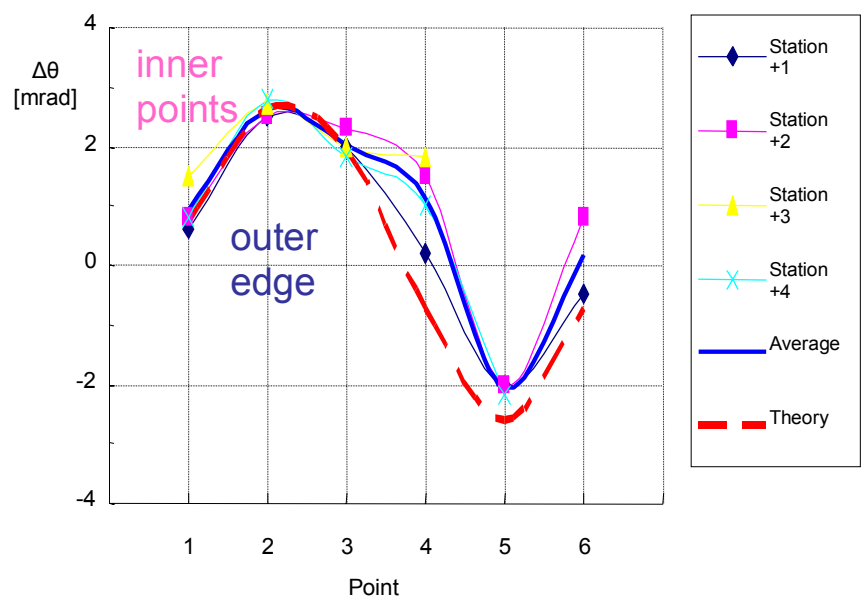

Fig. 12. Bending angle for stations $\mathrm{ME}+1,+2,+3,+4$ at six points around the outer edges of the disks for $\mathrm{B}=4.0 \mathrm{~T}$. 


\section{E. Proximity Sensor Results}

The change in azimuthal distance between two representative outer $\mathrm{ME}+1$ chamber is shown as a function of central magnetic field in Fig. 14. The distance increases quadratically with magnetic field and is on the order of 600 to $700 \mu \mathrm{m}$ at full field. The observed increase in distance between all chamber implies that the section of the face of $\mathrm{YE}+1$ at the outer ring of chambers is expanding azimuthally with increasing magnetic field.

\section{F. Disk Deformation: Data vs. Finite Element Analysis}

The understanding of chamber displacements due to endcap yoke disk deformations that emerges from the results and observations presented above can be summarized with the sketch shown in Fig. 15. Due to the strong gradient in the magnetic field near the end of the solenoid, strong magnetic forces pull the central portions of the endcap disks towards the solenoid. The various $Z$-stops, which prevent the disks from getting pushed into each other and onto the Muon Barrel, cause the endcap disks to bend into a cone shape.

The particular positions of the Z-stops between endcap and barrel near half the radius cause the side of the $\mathrm{YE}+1$ disk facing the barrel to compress radially around the Z-stops while expanding azimuthally. This explains the radial compression

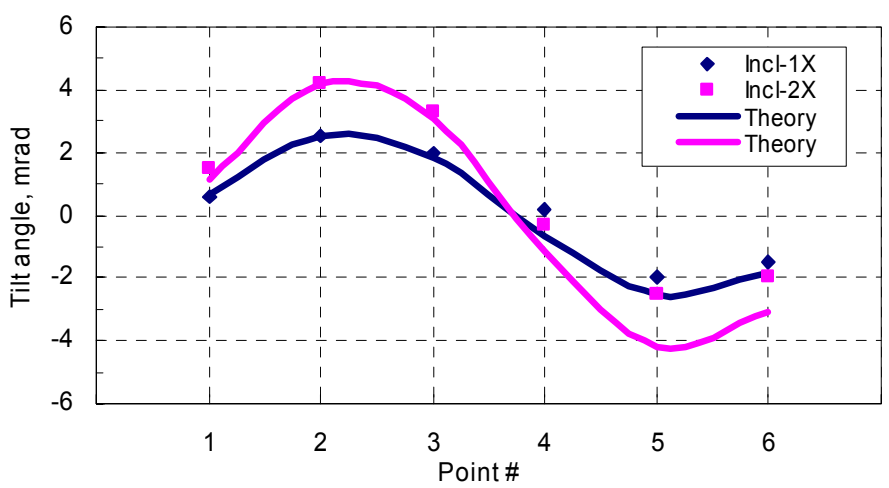

Fig. 13. Bending angle for station $\mathrm{ME}+1$ at six points around the outer edge (Incl-1X, blue) and at mid-radius between the outer and middle rings of chambers (Incl.-2X, magenta).

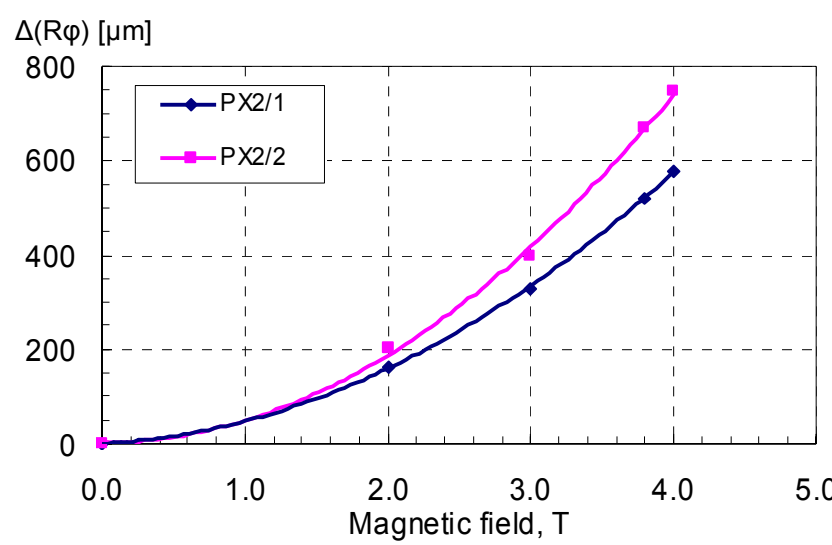

Fig. 14. Change in $R \varphi$-distance between two of the non-overlapping $\mathrm{ME}+1$ chambers vs. magnetic field as measured by two representative proximity sensors. of the face of $\mathrm{ME}+1$ and the bending angles at mid-radius of $\mathrm{ME}+1$ that are larger than at the outer edge.

Endcap disk deformations as predicted by a Finite Element Analysis using the ANSYS program for the CMS Muon Technical Design Report [1] are shown in Fig. 16. The color code indicates a predicted back bending in the $+\mathrm{z}$ direction (red) away from the barrel at the outer perimeter and bending

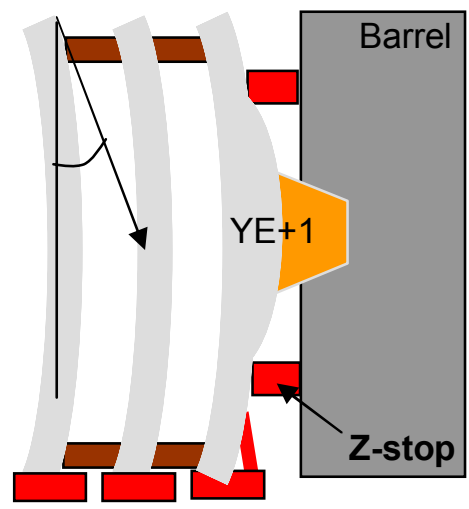

Fig. 15. Current understanding of disk deformation due to magnetic forces based on analog sensor measurements. The Z-stops (red) prevent the disks from getting pushed into each other. Note that the indicated bending angle is exaggerated for illustrative purposes.

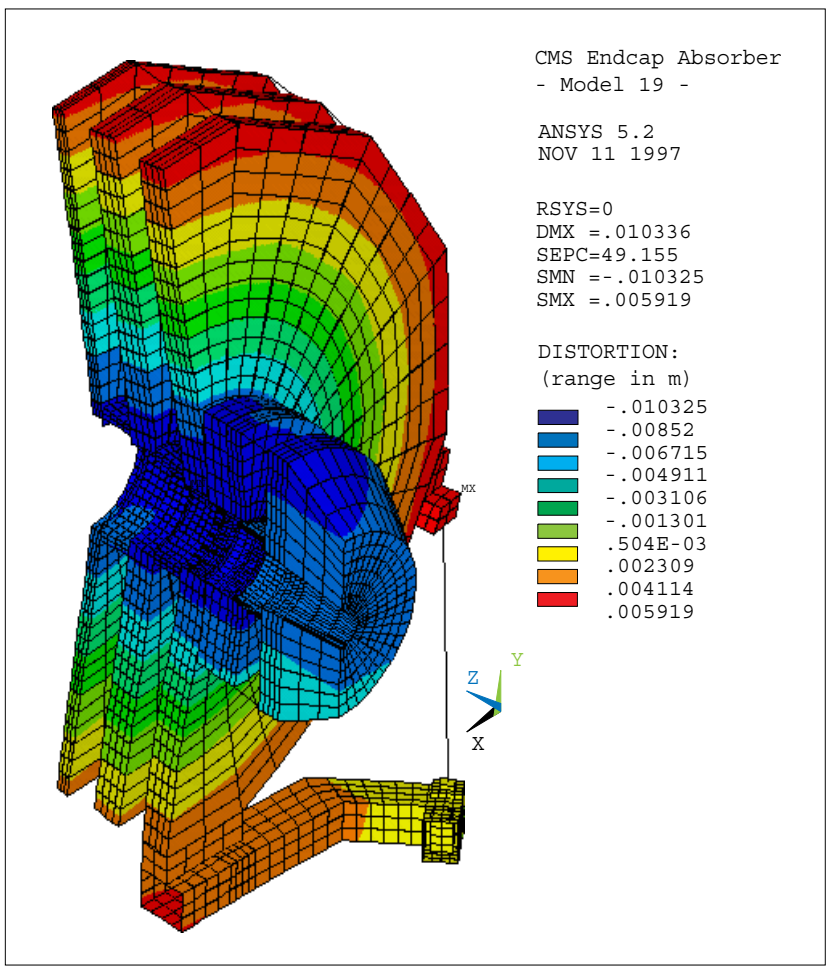

Fig. 16. Calculated deformation in the z-direction of the Muon Endcap return yoke due to the $4 \mathrm{~T}$ magnetic field. The inner edges of the endcap disks were predicted to move $\sim 10 \mathrm{~mm}$ toward the IP while the outer edges were predicted to move $\sim 6 \mathrm{~mm}$ away from the IP. The cart supports for disks YE2 and YE3 are not shown. YE+1 Z-stops were not included in the analysis. 
in $-\mathrm{Z}$ direction (blue) towards the barrel in the inner part of the endcap. The back bending is stronger at the top than at the bottom due to the carts that support the disks.

A final quantitative comparison of the $\mathrm{YE}+1$ disk deformation measured with the alignment sensors and the FEA prediction is shown in Fig. 17 in form of an RZ cross section across the $\mathrm{ME}+1$ disk. Reasonable quantitative agreement is found for all displacements and front face tilts. The front Z-stops were not included in the FEA, which explains that their effects appear in the data but not in the FEA prediction.
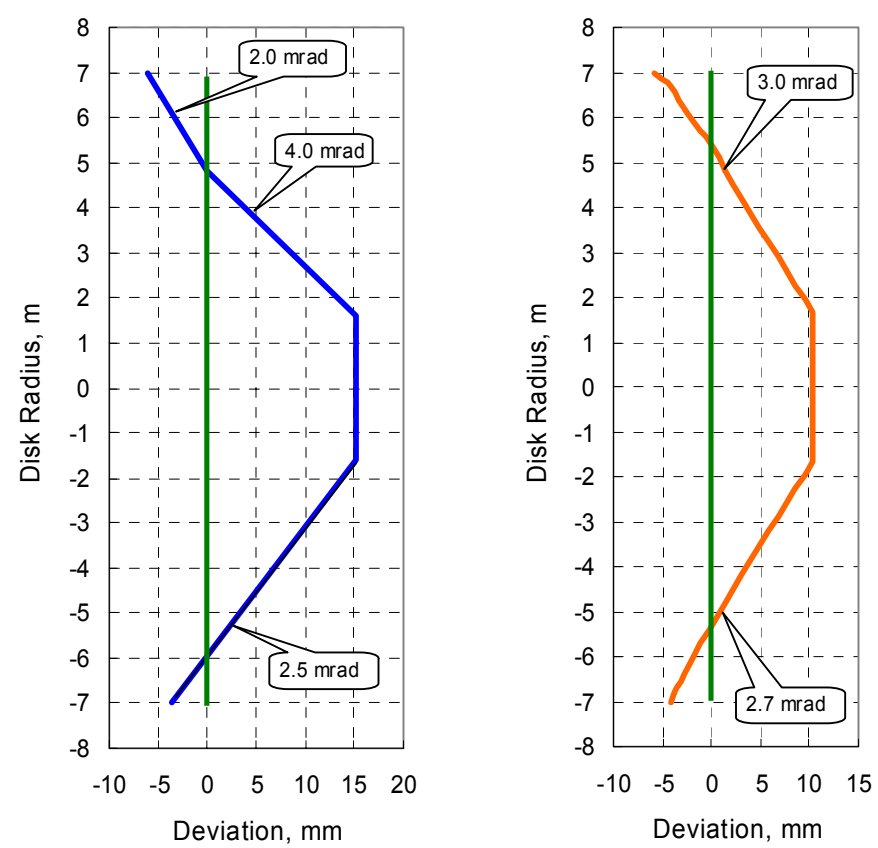

Fig. 17. Comparison of disk deformations in the RZ plane at full magnetic field (4T) measured with analog sensors (left) and predictions from Finite Element Analysis (right).

\section{REFERENCES}

[1] CMS: The Muon Project. Technical Design Report, CERN Report LHCC 97-32, 1997.

[2] CMS: The Magnet Project. Technical Design Report, CERN Report LHCC 97-10, 1997.

[3] R. H. Lee, "Simulation and Study of the CMS Endcap Muon Alignment Scheme," Ph.D. thesis, Purdue University, 2002.

[4] J. Moromisato, S.Reucroft, R. Terry, E. von Goeler, D. Eartly, and K. Maeshima, "The development of totally transparent position sensors," Nucl. Instr. and Meth. A, vol. 538, pp. 234-242, 2005.

[5] M. Ripert, "Calibration of Analog Sensors for the Alignment of Muon Chambers in the CMS experiment," M.S. thesis, Florida Institute of Technology, 2005. 